\title{
Meta
}

Journal des traducteurs

Translators' Journal

\section{Caignon, Philippe (2000) : Essential Lexicon in Accounting, coll. Champs linguistiques, Fides, Saint-Laurent (Quélbec), 197 p.}

\section{Jean Quirion}

Volume 47, numéro 1, mars 2002

URI : https://id.erudit.org/iderudit/007996ar

DOI : https://doi.org/10.7202/007996ar

Aller au sommaire du numéro

Éditeur(s)

Les Presses de l'Université de Montréal

ISSN

0026-0452 (imprimé)

1492-1421 (numérique)

Découvrir la revue

Citer ce compte rendu

Quirion, J. (2002). Compte rendu de [Caignon, Philippe (2000) : Essential Lexicon in Accounting, coll. Champs linguistiques, Fides, Saint-Laurent (Québec), 197 p.]

Meta, 47(1), 125-128. https://doi.org/10.7202/007996ar

Ce document est protégé par la loi sur le droit d'auteur. L'utilisation des services d’Érudit (y compris la reproduction) est assujettie à sa politique d'utilisation que vous pouvez consulter en ligne.

https://apropos.erudit.org/fr/usagers/politique-dutilisation/
Cet article est diffusé et préservé par Érudit.

Érudit est un consortium interuniversitaire sans but lucratif composé de l’Université de Montréal, l’Université Laval et l’Université du Québec à Montréal. Il a pour mission la promotion et la valorisation de la recherche. https://www.erudit.org/fr/ 


\section{DOCUMENTATION}

\section{Comptes rendus}

Caignon, Philippe (2000): Essential Lexicon in Accounting, coll. Champs linguistiques, Fides, Saint-Laurent (Québec), 197 p.

La phraséologie a pris une place grandissante dans le discours des théoriciens et des praticiens de la terminologie depuis plusieurs années. À peu près tous conviennent de l'importance de recourir à des vocables cooccurrents afin d'exprimer un message avec précision et clarté. Cependant, les répertoires terminographiques, papier ou électroniques, tardent à donner à la phraséologie la place que les langagiers affirment qui lui revient. En effet, qu'ils prennent la forme de gigantesques bases de données (comme TERMIUM et le Grand dictionnaire terminologique) ou d'un simple vocabulaire papier, ces répertoires réduisent encore généralement sa présence à la portion congrue.

En revanche, on constate parfois la publication de lexiques ou de vocabulaires mettant nettement l'accent sur la recension des cooccurrents et phraséologismes. Or, un ouvrage de ce type vient de paraître: Essential Lexicon in Accounting de Philippe Caignon. Ce dernier s'est joint au département d'Études françaises de l'Université Concordia, à titre de professeur de terminologie et de traduction. Sa thèse de doctorat, soutenue en 1996, portait sur la description de la langue de la finance. L'auteur prépare à l'heure actuelle un vocabulaire français (avec équivalents anglais) de termes comptables utilisés au Canada. Caignon a déjà publié, en 1995, le Dictionnaire anglais-français et français-anglais des biotechnologies, en collaboration avec Amal Jammal.

L’ouvrage est un vocabulaire anglais avec équivalents français. Un index françaisanglais figure à la fin du livre. Les définitions sont rédigées en anglais et les cooccurrents et phraséologismes ne s'appliquent qu'aux entrées anglaises. On peut estimer à 550 entrées la nomenclature du répertoire, qui inclut abréviations et variantes orthographiques.

L'article est organisé de façon classique. La catégorie grammaticale et, le cas échéant, le genre accompagnent chacune des entrées. L'article comprend toujours une définition anglaise, laquelle peut être suivie de synonymes anglais. Viennent ensuite les équivalents français, puis des notes sur un des termes ou sur la notion. L'article se termine sur l'énumération des cooccurrents et de la phraséologie. Ces informations sont catégorisées en nom, verbe et adjectif. Par exemple, pour l'entrée account, on propose entre autres:

nouns: name of an account

verbs: to affect an account

adjectives: comparable account

L'ouvrage s'adresse aux traducteurs, rédacteurs et réviseurs professionnels, ainsi qu'aux étudiants et aux professeurs de traduction et de comptabilité. L'auteur n'expose 
pas la façon dont l'ouvrage a été conçu et n'inclut pas d'illustrations ou de tableaux qui appuieraient les explications notionnelles.

Le répertoire comprend une bibliographie de 67 notices, essentiellement canadiennes, assez également réparties entre les sources anglaises et françaises. Les références sont généralement récentes, quatre sources sur cinq remontant à moins de dix ans. On note quelques coquilles, dont une adresse Internet incomplète, et le recours à des dictionnaires généraux dont il existait déjà, au moment de la préparation de l'ouvrage, des versions plus récentes.

La maison d'édition, Fides, publie régulièrement des ouvrages de référence, mais sa présence dans le domaine terminologique est plutôt restreinte. L'ouvrage s'inscrit dans la collection Champs linguistiques, qui «publie des ouvrages portant sur tous les aspects de la linguistique [...] et des ouvrages portant sur l'interaction entre la linguistique et d'autres disciplines ", dont la traduction.

L'auteur annonce, dans sa courte présentation, que les entrées répertoriées incluent les notions fondamentales de la comptabilité. Nous avons tenté de déterminer la part de nouveauté contenue dans la macrostructure de Essential Lexicon in Accounting. Nous avons pour ce faire sélectionné au hasard $10 \%$ des entrées de l'ouvrage: la première entrée apparaissant au haut d'une page, toutes les trois pages, a fait l'objet d'une interrogation dans TERMIUM. Il en ressort que 42 des 54 termes interrogés y font l'objet d'une fiche dans le domaine de la comptabilité ou dans un domaine connexe. Pour arriver à ce résultat, nous avons comparé les informations sur les fiches avec les définitions présentées par Caignon. Neuf autres termes sont absents de TERMIUM, bien que la banque fédérale consacre une fiche à la notion qu'ils désignent ; c'est le cas, par exemple, de double entry, synonyme de double entry bookkeeping. Le premier est absent de TERMIUM, alors que le deuxième y figure. On constate donc que Caignon recense plusieurs synonymes absents de TERMIUM. Enfin, deux autres entrées sont uniques à l'ouvrage et une dernière (l'adjectif business, rendu par commercial) est sans doute trop générale pour figurer dans TERMIUM. On peut donc confirmer, à partie de cette rapide étude, que l'originalité de l'ouvrage de Caignon ne réside pas dans la nouveauté des entrées qu'il traite, mais bien dans les cooccurrents et phraséologismes qu'il recense. On en compte plus d'une centaine à l'entrée company, mine d'or pour une expression juste.

On remarque qu'un peu plus de clarté dans l'organisation de l'ouvrage permettrait d'en faire une meilleure utilisation. Par exemple, une entrée synonymique apparaissant à la nomenclature renvoie à l'entrée principale où est traité le terme. Des cooccurrents et phraséologismes figurent néanmoins sous l'entrée synonymique. Bien que ce ne soit mentionné nulle part, on comprend que ces informations sont propres au synonyme. Corollairement, cela signifie qu'à l'entrée principale, les cooccurrents ne valent que pour cette dernière, ce que l'auteur ne précise pas. Autre exemple: on peut reprocher à l'auteur son classement alphabétique des équivalents français de chaque article. En effet, on déduit que les termes français ne sont pas présentés en ordre de fréquence, de préférence ou d'importance. Ainsi, pour l'entrée anglaise auditor, on trouve audit comme premier de cinq équivalents français. Mais une note subséquente indique que son emploi est déconseillé. Le lecteur doit donc lire la totalité des entrées françaises chaque fois qu'il consulte un article, puis procéder au choix de l'équivalent au meilleur de ses connaissances. Il en est de même pour les synonymes 
anglais; seul le choix de l'entrée principale anglaise ne semble pas reposer sur l'ordre alphabétique, mais sur un choix logique.

Des renvois antonymiques sont faits à l'occasion entre certaines notions. Ils ne sont cependant pas toujours réciproques; par exemple, une note sous double entry bookkeeping signale que cette notion s'oppose à celle de single entry bookkeeping, mais le renvoi n'est pas fait dans l'autre direction.

L'auteur affirme en introduction tenir compte des variations géographiques et marquer les usages américains, australiens, belges, britanniques, canadiens, français, ainsi que ceux de la Communauté européenne ${ }^{1}$. Une absence à cette liste est celle des usages québécois; on en retrouve pourtant mention dans quelques entrées, dont business corporation, pour qualifier compagnie et compagnie à fonds social.

Le recours ambivalent de l'auteur aux dictionnaires généraux est déroutant. Il suggère par exemple que le terme français audit, qu'il dit présent dans des dictionnaires généraux, soit rejeté à cause de son absence des sources spécialisées. Par contre, à l'entrée fiscal year, il appuie des affirmations sur des dictionnaires généraux. Il précise que, selon le Petit Robert, exercice financier ne correspond pas exactement à fiscal year et que, selon le Multidictionnaire des difficultés de la langue française, année budgétaire et année financière sont utilisés uniquement dans la comptabilité du secteur public.

Les notes sont nombreuses et les informations proposées sont pertinentes. Il est étonnant cependant de constater que les formes féminines de titres français sont mentionnées en note au lieu d'apparaître avec les autres équivalents. Par ailleurs, ce ne sont pas tous les titres de fonction qui voient leur forme féminine mentionnée.

On peut regretter l'absence de lettrine au haut de chaque page, qui aurait l'avantage d'accélérer la consultation. Celle-ci est d'autant plus ralentie que le haut d'une page renferme souvent la fin d'une entrée commencée à la page précédente et que la présentation typographique de l'article rend malaisée la localisation rapide de l'entrée suivante.

Il est gênant de noter la présence d'homonymes non numérotés. Par exemple, quatre entrées goods sont présentées les unes à la suite des autres, sans distinction. Pourtant, l'index français-anglais renvoie à des numéros (par exemple, produits (1) renvoie à goods (4)). Il appartient donc au lecteur de les numéroter ou de les compter chaque fois; une erreur est alors facile à commettre et un équivalent inapproprié ou une cooccurrence maladroite risquent d'en résulter.

Autre danger de confusion : à plusieurs endroits, les formes abrégées de syntagmes (SA pour société anonyme, par exemple) portent les mêmes marques d'usage topolectales (ici, Belgique, France) que les formes au long, mais les appels de note figurent seulement à la forme complète. Les notes en question précisent la définition, donnent des explications sur la notion, ici en fonction des contextes belges et français. Comme le classement des équivalents est alphabétique, les abréviations et les formes au long ne se suivent pas dans la liste; des notes utiles au lecteur peuvent donc facilement lui échapper.

Certaines entrées sont au pluriel sans raison évidente. En outre, des synonymes renverront à des entrées au singulier, qui apparaîtront en fait au pluriel dans la nomenclature (accumulated tax allocation renvoie à deferred income tax; la nomenclature affiche plutôt deferred income taxes); par ailleurs, TERMIUM consigne ces entrées au 
singulier. De plus, l'auteur ne précise pas, dans les informations grammaticales, le statut pluriel de certaines entrées.

En conclusion, malgré certains défauts, Essential Lexicon in Accounting est un répertoire utile. Non pas tant parce qu'il présente les notions fondamentales de la comptabilité, ainsi que les termes anglais et français qui les désignent; d'autres l'ont fait avant lui. Son originalité tire plutôt sa source dans l'intégration des cooccurrents et des phraséologismes propres à l'anglais du domaine. D'une grande richesse, ces informations sauront être mises à profit par le public visé par le répertoire, soit les professionnels et étudiants de traduction et de comptabilité.

JEAN QUIRION

Université du Québec à Hull, Hull, Canada

\section{NOTES}

1. On peut se demander si l'auteur veut vraiment faire référence à la Communauté européenne (nouveau nom du Conseil des ministres de la Communauté européenne) ou s'il ne réfère vraisemblablement pas plutôt à l'Union européenne (nouveau nom, depuis 1993, de la Communauté européenne).

\section{RÉFÉRENCES}

Canada. Bureau de la traduction. Termium Plus, [En ligne], Ottawa, Travaux publics et Services gouvernementaux Canada, http://www.termiumplus.bureaudelatraduction.gc.ca/tpv2Show/ termiumplus.html ?lang $=\mathrm{f} 2$

Cormier, M. C. (1989): «L'élaboration de produits terminologiques dans les domaines de pointe: quelques constats », dans Meta, 34, 3, p. 586-593.

Pym, Anthony (2000): Negotiating the Frontier. Translators and Intercultures in Hispanic History, Manchester, St. Jerome Publishing, ix-265 p.

L'auteur nous fait faire une incursion dans le monde de la traduction qui s'échelonne sur près de neuf siècles. Même s'il situe la scène dans la sphère hispanique, les interrelations et les interactions, tant des acteurs que des metteurs en scène ou encore des conséquences qui en découlent, se reflètent sur l'ensemble du monde. La traduction, on le sait, peut être un puissant catalyseur de connaissances et de développement scientifique, technique, économique, politique, artistique, littéraire ou culturel et social et même linguistique. Après avoir noté dans son Introduction la valeur des interrelations culturelles tant conflictuelles que recouvrantes pour l'histoire du monde hispanique, l'auteur met en évidence le rôle de certains promoteurs et acteurs de 1142 à nos jours en montrant que certains faits contemporains plongent leurs racines dans le passé. Si le livre est riche en anecdotes et en paradoxes, ce ne sont que des exemples dont le but principal est d'illustrer des traits plus généraux et plus universaux. Le livre est divisé en douze chapitres qui s'intitulent: 1. The Abbot's Gold, 2. Toledo and All That, 3 The Price of Alfonso's Learning, 4. The Importance of Paper, 5. A Christian's Rabbinic Bible, 6. From translad- to traduc-, 7. The Language Empire, 8. The Language of Exile, 9. A Volcano Unbaptized, 10. Autorship in Translation Anthologies, 11.The Symbolic Olympics, 12. Training for Globalizing Markets. 